\title{
Green Protocols for the One-Pot Synthesis of Vanillin Based aminoalkyl and Amidoalkyl Naphthols and Their Antibacterial Activity
}

\author{
AFINISHA DEEPAM ${ }^{1 *}$ and JYOTHI VISWANADHAN ${ }^{1}$ \\ Department of chemistry, Christian college, Kattakada, Thiruvananthapuram, Kerala, India. \\ ${ }^{*}$ Corresponding Author: afinishadpm @gmail.com \\ http://dx.doi.org/10.13005/ojc/330336
}

(Received: February 06, 2017; Accepted: May 05, 2017)

\begin{abstract}
Environment-friendly, facile, cost effective green chemical methods are reported for the onepot multicomponent solvent less synthesis of 1-aminoalkyl-2-naphthol and 1-amidoalkyl-2-naphthol exploring tannic acid as a novel, biodegradable, cheap and efficient Lewis acid catalyst. Present investigation focuses on green chemistry approach for the synthesis of aminoalkyl and amidoalkyl compounds. A mixture of sufficient amount of tannic acid, vanillin, 2-naphthol and 4-nitroaniline/ benzamide is refluxed by following various green protocols such as oil bath, microwave irradiation, hot plate with magnetic stirrer, Grindstone method and finally conventional heating method. Structure of the products confirmed by spectroscopic techniques such as IR, ${ }^{1} \mathrm{H}$ NMR, ${ }^{13} \mathrm{C}$ NMR and GC-MS. Anti-bacterial activity of synthesized compounds against Bacillus subtilis is tested by zone inhibition method.
\end{abstract}

Keywords: Amidoalkyl naphthol, Aminoalkyl naphthol, microwave synthesis, Grindstone chemistry, Oil bath, Vanillin, 2-naphthol.

\section{INTRODUCTION}

Green chemistry is defined as sustainable chemistry, which is dealing with environment- friendly chemical synthesis. It is the design of chemical products and processes that reduce or eliminate the use of hazardous substances. Green chemistry covers the entire life cycle of a chemical product and aims to reduce hazardous substances from the design process, manufacturing, use, and final disposal. Solvents using in industry and volatile organic compounds (VOCs) lead to environmental pollution and risks to human health. One of the important green pathway to reduce pollution is solvent less synthesis; mainly in the field of organic chemistry. The toxicity and volatile nature of many organic solvents, particularly chlorinated hydrocarbons that are widely used in huge amounts for organic reactions have posed a serious threat to the environment. Thus, design of solvent less catalytic 
reaction has received tremendous attention in recent times. The drive for the development of dry media reactions in chemistry is the method is economics (save money on solvent), to avoid removal of a solvent after reaction completion, reaction rate is high due to more availability of reactants, environmentally friendly because solvent is not required and simple to handle. Solvent-less reactions can be successfully performed in the case of multicomponent reactions. One pot multicomponent reactions (MCR), sometimes called as zipper reactions have gained wide attention in the field of synthetic organic chemistry as they increase the efficiency of the reaction and decrease the number of laboratory operations along with quantities of solvent and chemicals used. They also reduce the reaction time considerably and facilitate the yield of products than the normal multiple step methods. MCR link several transformations in a single step. MCR is an important tool in reactions such as Biginelli, Ugi, Mannich and Betti reactions. The Betti reaction is an addition reaction between aldehydes, primary aromatic amines and phenols producing á-aminobenzylphenols. The product of the Betti reaction is called the Betti base. Betti bases are said to be aminoarylphenols.

1-amidoalkyl-2-naphthols are important compounds in the field of synthetic organic chemistry because it is building block for the synthesis of many vital compounds. There are remarkable intermediates which can be easily converted into biologically active 1-aminoalkyl-2- naphthol derivatives by amide hydrolysis. The hypotensive and bradycardiac effects of these compounds have been evaluated ${ }^{1}$. Also, amidoalkyl naphthols can be used for the synthesis of highly functionalized oxazine derivatives through Vilsmeier cyclisation reaction². In Vilsmeier cyclisation reaction, the oxazine derivatives are prepared from corresponding amidoalkyl naphthols by treating it with Vilsmeier reagent at room temperature. The reagent is a mixture of 12 equivalence of DMF (Dimethylformamide) and 8 equivalence $\mathrm{POCl} 3$. This experiment is based on the mechanism of Vilsmeier- Haack reaction which involves the synthesis of functionalized heterocycles. Molecules having 1, 3- oxazine moiety is important due to their large spectrum of pharmacological applications such as anti-tumor ${ }^{3}$, anti-malarial and anti-microbialactivities ${ }^{4}$. Because of its wide range of applications, synthesis of amidoalkyl naphthol derivatives has attracted the attention of chemists and various improved procedures have been reported.

Bronsted or Lewis acid catalysts have been utilized for the preparation of 1- amidoalkyl2-naphthols. This type of compounds can be prepared by the condensation of aromatic aldehydes, 2-naphthol and amides, urea or acetonitrile in the presence of a Lewis or Bronsted acid catalysts. Several methods have been documented in the literature for synthesis of these compounds using a variety of catalysts such as Montmorillonite K10 clay ${ }^{5} \mathrm{Ce}\left(\mathrm{SO}_{4}\right)_{2}{ }^{6}$, benzimidazolium based ionic liquid", Butyl methyl imidazolium bromide ${ }^{8}, \mathrm{~N}, \mathrm{~N}, \mathrm{~N}^{\prime}, \mathrm{N}^{\prime}$ Tetrabromobenzene- 1,3-disulfonamide (TBBDA) ${ }^{9}$, Carbon based solid $\operatorname{acid}^{10}$, Pentafluorophenyl ammoniumTriflate (PFPAT) ${ }^{11}$, Molten tetraethyl ammonium chloride ${ }^{12}$, Boric acid ${ }^{13}$, polyphosphate ester ${ }^{14}$ etc. but majority of these reactions need high temperature, long time, expensive reagents, high catalyst loading, corrosive reagents, strongly acidic conditions and low yields of products. Therefore introduction of a suitable ecofriendly catalyst and efficient methods for the preparation of 1-amidoalkyl2-naphthols and 1-aminoalkyl-2-naphthols are of practical importance.

Tannic acid, a naturally occurring plant polyphenol is used as the mild Lewis acid catalyst for the synthesis of aminoalkylnaphthols, amidoalkylnaphthols ${ }^{15}$ and some oxazine derivatives. The chemical formula for commercial tannic acid is given as $\mathrm{C}_{76} \mathrm{H}_{52} \mathrm{O}_{46}$, i.e., a molecule with deca-galloyl glucose. (Gallic acid ester of glucose).

But sometimes exists as a mixture of polygalloyl glucoses or polygalloylquinic acid esters with the number of galloyl moieties per molecule ranging from 2 up to 12 . It is depending on the plant source used to extract the tannic acid. Tannic acid contains central glucose molecule derivatised at its hydroxyl groups, with one or more galloyl residues. Its weak acidity (pKa around 10) is due to the numerous phenol groups in the structure. Tannic acid exists in two forms. Quercitannic acid found in oak bark and leaves and Gallotannic acid found in oak galls. Molar mass of tannic acid is $1701.19 \mathrm{~g} / \mathrm{mol}$ and is highly soluble in water $(2850 \mathrm{~g} / \mathrm{L})$. Commercial tannic acid is usually extracted from plant parts such as Caesalpinia 
spinosa (commonly known as Tara pods or Quechua is a small leguminous tree or thorny shrub native to Peru), gallnuts from Rhussemialata (Chinese sumac or nutgall tree) or Quercusinfectoria(a species of oak, bearing galls that have been traditionally used in Asia as medicine).

Strong acids cannot be used for catalyzing multicomponent organic synthesis which involve aldehydes. Because use of acidic catalysts in aldehydes bearing basic groups or acid sensitive aldehydes is not possible. Polyphenolic nature of tannic acid may be explored for their catalytic efficiency. Tannic acid is successfully used as mild Lewis acid catalyst in the Betti type multicomponent synthesis of amidoalkylnaphthols ${ }^{15}$ and 2, 4, 5-triaryliH-imidazole ${ }^{16}$.
Solvent less synthesis of amidoalky and aminoalkyl naphthols have been done by adopting various methods such as microwave irradiation, heating on an oil bath, heating on hot plate with magnetic stirring and Grindstone chemistry method ${ }^{17}$.As already mentioned, various pharmaceutical applications of amidoalkyl naphthols as well as aminoalkyl naphthols are reported. Verma et al., reported the synthesis of 1, 3- disubstituted$1 \mathrm{H}$-naphthol [1, 2-e] [1, 3] oxazines and evaluation of in vitro antibacterial activity against Gram-positive Bacillus subtillis [MTCC 2063], Staphylococcus aureus [MTCC 2901] and Gram-negative $E^{18}$. coli[MTCC 8184]. These compounds are synthesized from amidoalkylnaphthols. Also, antibacterial activities of some amidoalkylnaphthols (synthesized using nano- $\mathrm{TiSiO} 2$ as catalyst) against standard

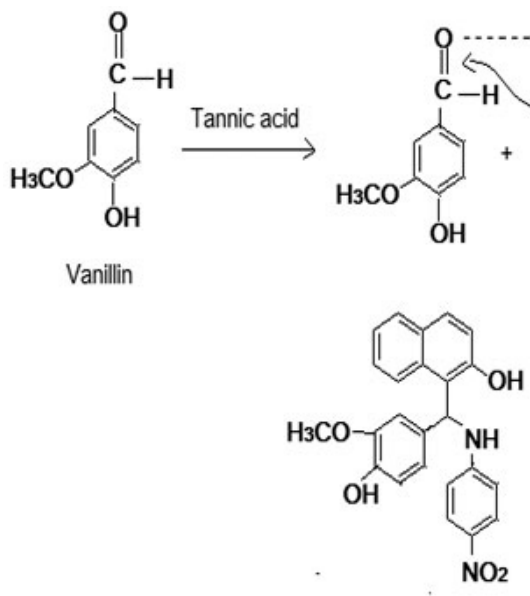

Product A

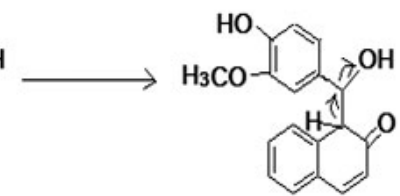

2-naphthol

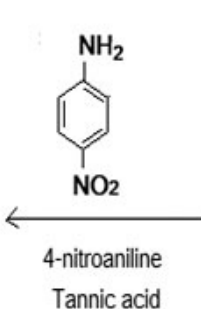

Tannic acid

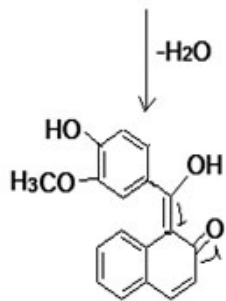

o-QM

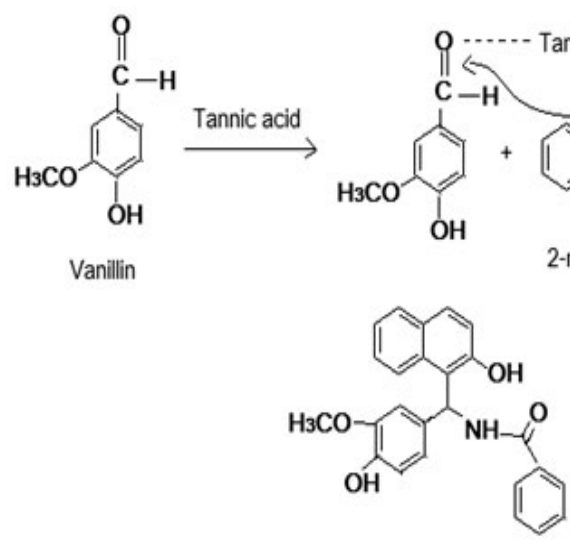

Product B<smiles>COc1ccc(OC(=O)C2C(=O)C=Cc3ccccc32)cc1O</smiles><smiles>NC(=O)c1ccccc1</smiles><smiles>COc1cc(C(O)=C2CC3(CC=O)C=CC(C2=O)c2ccccc23)cc(O)c1O</smiles>

o-QM

Mechanism of reactions and structure of products 
species of S. aureus [MTCC 25293], En.Faecalis [MTCC 11700] and P. aeruginosa [MTCC 27853] are discovered by ${ }^{19}$.Synthesized compounds are tested for their anti-bacterial activity using zone inhibition method. Zone Inhibition Test, also called a Kirby-Bauer Test, is a qualitative method used clinically to measure antibiotic resistance and industrially to test the ability of solids and textiles to inhibit microbial growth. Researchers who develop antimicrobial textiles, surfaces, and liquids use this test as a quick and easy way to measure and compare levels of inhibitory activity.

In this work, we synthesized and characterized one compound from each type of the compounds aminoalkyl and amidoalkylnaphthols through different methods such as oil bath, microwave irradiation, hot plate with magnetic stirrer and Grindstone chemistry method. The reactants used are aromatic aldehyde (vanillin), 2- naphthol and aromatic amine (4-nitro aniline) or aromatic amide (benzamide) for getting aminoalkyl and amidoalkylnaphthols respectively. Also, the two compounds are tested for their anti-bacterial

Table 1: $R_{F}$ Values of Reactants in a Solvent System of Hexane: Ethyl acetate (4:1)

\begin{tabular}{lcc}
\hline No & Name of reactant & $\mathbf{R}_{\mathbf{f}}$ value \\
\hline 1 & 2-naphthol & 0.8064 \\
2 & Vanillin & 0.2129 \\
3 & 4-nitroaniline & 0.3870 \\
4 & Benzamide & 0.0903 \\
\hline
\end{tabular}

activity against Bacillus subtilis. It is known also as the hay bacillus or grass bacillus, a Gram-positive, catalase-positive bacterium, found in soil and the gastrointestinal tract of ruminants and humans.

\section{MATERIALS AND METHODS}

All chemicals and solvents were purchased from central scientific laboratory supplies, Panavila, Thiruvananthapuram. The melting points were determined by an open capillary method and are uncorrected. All chemicals used are of analar grade. Reactants used are 2-naphthol, vanillin, p-nitroaniline, benzamide and tannic acid. Solvents used are ethanol, chloroform, ethyl acetate, hexane, acetone, DMSO etc.

Synthesis of two compounds were done by following different methods using the naturally occurring plant polyphenol tannic acid. Compound $A$ is an aminoalkylnaphthol which is synthesized from 2-naphthol, vanillin and 4-nitroanilin and compound $\mathrm{B}$ is an amidoalkylnaphthol synthesized by the condensation of 2- naphthol, vanillin and benzamide.

Procedure for the synthesis of Aminoalkyl and AmidoalkyINaphthols via Different Methods

\section{Oil bath}

A mixture of 2-naphthol, vanillin and p-nitroaniline is taken in a ratio $1: 1: 1.3 \mathrm{mmol}$ (0.144: 0.152: 0.1795 ) and $0.02 \mathrm{mmol}$ of tannic acid $(0.03402 \mathrm{~g})$ mixed in a $100 \mathrm{ml}$ conical flask. In another

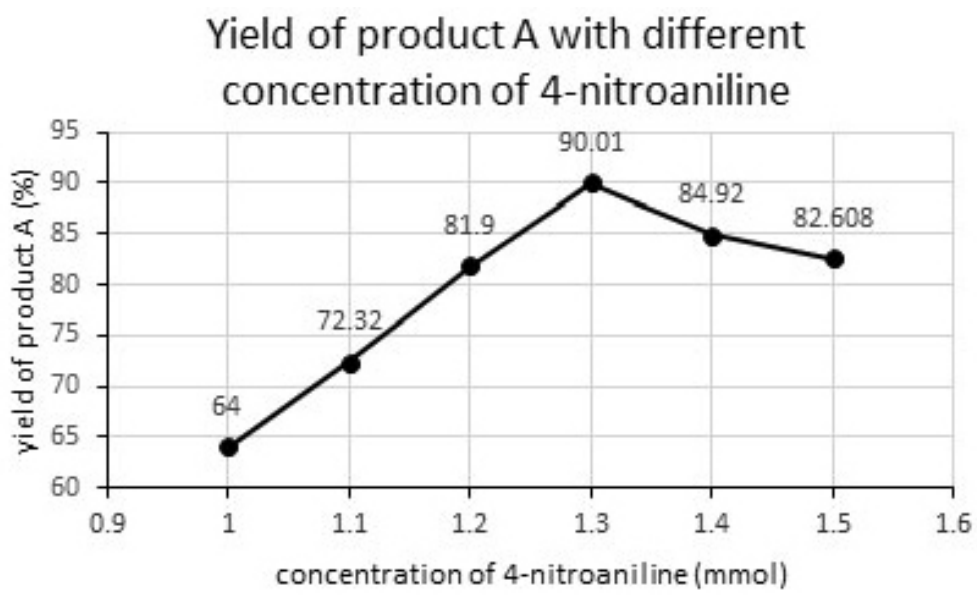

Fig 1: A plot of concentration of 4-nitroaniline against percentage of yield of product A 
conical flask, mixture of 2-naphthol, vanillin and benzamide (0.144: $0.152: 0.1574 \mathrm{~g}$ ) and $0.03 \mathrm{mmol}$ of tannic acid $(0.05103 \mathrm{~g})$ is taken. Both the reaction mixtures are heated in oil baths of cotton seed oil kept in hot plate at $120-1250 \mathrm{C}$ for $10-15 \mathrm{~min}$. To know whether the reaction is complete, samples are taken from the reaction mixture at constant intervals of time and TLC is performed in each case. After the completion of reaction, spot for the product is detected by performing TLC using the same solvent system (hexane: ethyl acetate-1:4) and $R f$ values are compared with those obtained for reactants. After completing the reaction, the solid form of crude product is cooled to room temperature, washed with water to remove water soluble unreacted vanillin and tannic acid. Finally, pure product is obtained through the recrystallization using ethanol (EtOH: $\mathrm{H} 2 \mathrm{O}-1: 3)^{20}$.

\section{Microwave irradiation}

Reaction mixture is 2- naphthol, vanillin, and p-nitroaniline (or benzamide) in the ratio 1:1:1.3 mmol added with $0.02 \mathrm{mmol}$ (or $0.03 \mathrm{mmol}$ ) of tannic acid and it was then placed in a microwave oven (Model no: MW73AD-B/XTL, SAMSUNG 800W), at $100 \mathrm{~W}$. Each pulse of $30 \mathrm{~s}$ with intermittent time is applied to avoid overheating. The time taken for completing the reaction is determined by performing the reaction for 1-4 $\mathrm{min}$. The completion of reaction checked by TLC. After completion of reaction, the

\section{Concentration of tannic acid and product $A$}

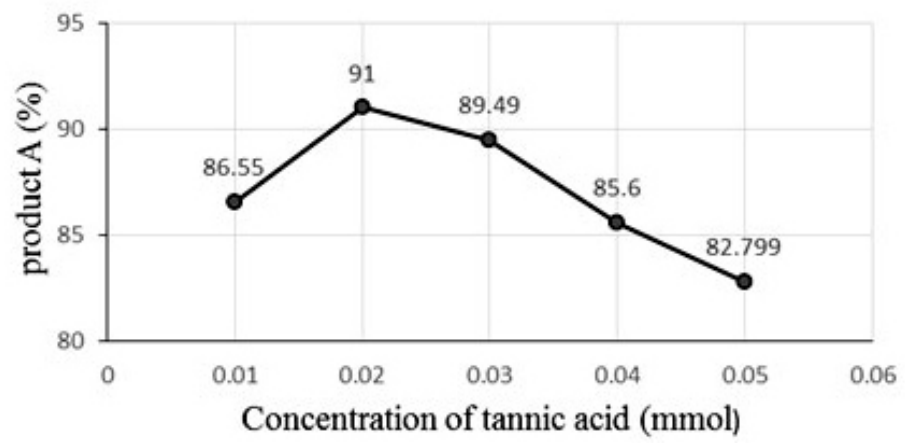

Fig 2: A plot of concentration of benzamide against percentage of yield of product B

Table 2: Determination of Ratio of Reactants to get Maximum Yields to get Products $a$ and $b$

\begin{tabular}{|c|c|c|c|}
\hline \multicolumn{3}{|c|}{$\begin{array}{l}\text { Amount of reactants (mmol) } \\
\text { Product A- aminoalkylnaphthol }\end{array}$} & \multirow[t]{2}{*}{ Yield of product $\mathbf{A}(\%)$} \\
\hline Vanillin & 2-naphthol & p-nitroaniline & \\
\hline 1 & 1 & 1 & 64 \\
\hline 1 & 1 & 1.1 & 72.32 \\
\hline 1 & 1 & 1.2 & 81.9 \\
\hline 1 & 1 & 1.3 & 90.01 \\
\hline 1 & 1 & 1.4 & 84.92 \\
\hline 1 & 1 & 1.5 & 82.608 \\
\hline \multicolumn{3}{|c|}{ Product B- amidoalkylnaphthol } & Yield of product B (\%) \\
\hline Vanillin & 2-naphthol & Benzamide & \\
\hline 1 & 1 & 1 & 76.9 \\
\hline 1 & 1 & 1.1 & 84.3 \\
\hline 1 & 1 & 1.2 & 87 \\
\hline 1 & 1 & 1.3 & 92.1 \\
\hline 1 & 1 & 1.4 & 89.76 \\
\hline 1 & 1 & 1.5 & 86.85 \\
\hline
\end{tabular}


mixture is cooled to room temperature and washed with cold water. Pure crystals recrystallized from the crude product using ethanol.

\section{Hot plate with magnetic stirrer}

A mixture of vanillin, 2-naphthol, and p-nitroaniline (or benzamide) in 1:1:1.3mmol is taken in a white marble mortar and pestle. It is grounded well with $0.02 \mathrm{mmol}(0.03 \mathrm{mmol})$ of tannic acid and transferred into a $100 \mathrm{ml}$ conical flask and stirred after keeping magnetic fish. The mixture is then heated in hot plate (REMI 2MLH) at about 120-125oC. The process is continued for $20-25$ min keeping the temperature constant and regulating the speed of stirring. The completion of the reaction is checked by TLC using the solvent system hexane: ethyl acetate (4:1). The resulting mass was cooled, extracted with water, and recrystallized from ethanol.

\section{Grindstone chemistry method}

A mixture of reactants (2-naphthol, vanillin, and $p$-nitroaniline (or benzamide) in the ratio 1:1:1.3 $\mathrm{mmol}$ is taken in marble mortar and finely ground using pestle for 10-15 min with catalyst, tannic acid powder $(0.02$ or $0.03 \mathrm{mmol})$. The reaction is checked by TLC, then the resultant mass was washed with water and the crystals of final product are obtained by recrystallization using ethanol.

\section{Normal Refluxing}

A mixture of reactants and catalyst in the same ratio as described above is refluxed with a few drops of methanol for 60-90 min. The reaction progress was followed by TLC. Pure product is separated by washing with water and recrystallization using ethanol.

\section{Yield of product B with different concentration of Benzamide}

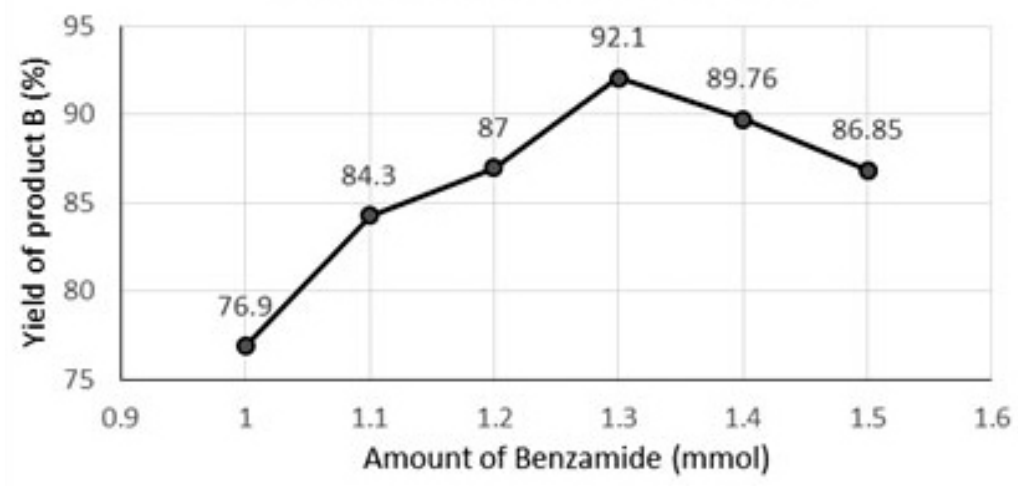

Fig 3: A plot of concentration of tannic acid against yield of product A

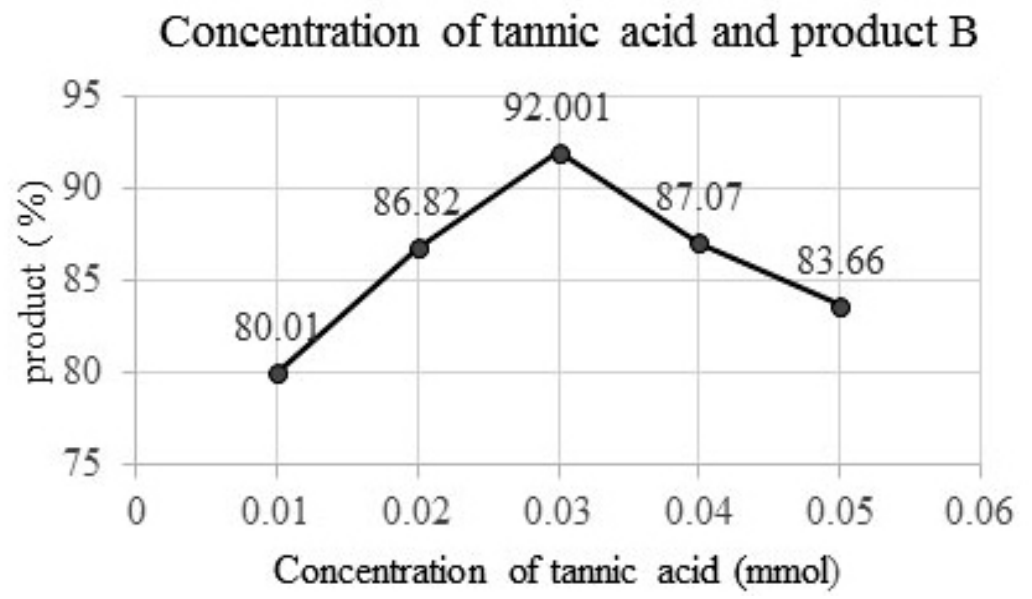

Fig 4: A plot of concentration of tannic acid against yield of product B 
Purification of products is done by column chromatography. The column is packed with silica gel (200-400). The crude product is dissolved in chloroform and loaded in the column. It is eluted with hexane: ethyl acetate of ratio $1: 1^{8}$. The eluted compounds are separated byTLC using solvent system Hexane: ethyl acetate (4:1) and developed spots were visualized after keeping in iodine chamber and $R_{f}$ value is noted.

Ultraviolet-Visible spectra of purified products are obtained by dissolving in ethanol and recorded in UV-Visible spectrometer (UV-Vis-NIR Spectrophotometer, Varian Cary 5000 with wavelength range $175-3300 \mathrm{~nm}$ ). IR spectra is recorded in IR spectrometer (Schimadzu IR prestige-20 spectrometer) with $\mathrm{KBr}$ as the reference compound and compared the values with that of reactants. The products were also analyzed using ${ }^{1} \mathrm{H}$ NMR, ${ }^{13} \mathrm{C}$ NMR (NMR spectrometer, BrukerAvance III, $400 \mathrm{MHz}$ ) and GC-MS in Thermo ExactiveOrbitrap (Gas Chromatography Mass spectrometer, Shimadzu 5050A-Model) techniques.

Table 3: Concentration of Tannic acid and the Yield of Products

\begin{tabular}{lcc}
\hline $\begin{array}{l}\text { Conc. } \\
\text { of catalyst, } \\
\text { tannic acid } \\
\text { (mmol) }\end{array}$ & \multicolumn{2}{c}{ Yield of products (\%) } \\
\cline { 2 - 3 } & Compound A & Compound B \\
\hline 0.01 & 86.55 & 80.01 \\
0.02 & 91.0 & 86.82 \\
0.03 & 89.49 & 92.001 \\
0.04 & 85.60 & 87.07 \\
0.05 & 82.799 & 83.66 \\
\hline
\end{tabular}<smiles>COc1cc(C(Nc2ccc([N+](=O)[O-])cc2)c2c(O)ccc3ccccc23)ccc1O</smiles>

Product A

Fig. 5: Structure and IUPAC name of product A.1-[(4-hydroxy-3-methoxyphenyl) (4-nitrophenylamino)] methyl naphthalene-2-ol
The two products $A$ and $B$ are subjected to zone inhibition test to study about their anti- bacterial activity. A culture of bacterium Bacillus subtilis is allowed to grow in a petri plate in agar medium. Using a sterile swab, a suspension of the pure culture is spread evenly over the face of a sterile agar plate. It is stored 24-48 $\mathrm{h}$ for attaining normal bacterial growth. The compounds are introduced into the holes bored in the plate and incubated overnight. Size of the zone visible around the hole is measured.

\section{RESULTS AND DISCUSSION}

To study the synthesis of amidoalkyl and aminoalkylnaphthols, Betti reaction is considered as a standard reaction. Betti reaction is the condensation reaction of aromatic aldehydes, primary aromatic or heterocyclic amines and phenols leading to $\alpha$-aminobenzylphenols.

Compound A: Aminoalkylnaphthol, which is prepared by the condensation of 2-naphthol, vanillin and 4-nitroaniline in presence of tannic acid as catalyst.

Compound B: Amidoalkylnaphthol, which is prepared by the condensation of 2-naphthol, vanillin, and benzamide in presence of tannic acid as catalyst.

Monitoring reaction progress and detection of formed products were done using TLC and $R_{t}$ values are calculated. This data can be taken as the primary awareness about our products. The $R_{f}$ values of reactants are determined by conducting TLC of each of them in the solvent system, hexane: ethyl acetate (4:1). Rf values are shown in Table 1.

TLC of purified products and reactants were conducted in the above solvent system and compared $R_{f}$ values with that of reactants and new bands are identified viz: Product $A\left(R_{f}=0.4\right)$ and product $B\left(R_{f}=0.74\right)$. The optimization study of reactants is determined by keeping amount of 2-naphthol and vanillin as constant and varying third constituent (4-nitroaniline or benzamide). The reaction is done by refluxing in cotton seed oil bath. Results are tabulated in Table 2.

From the table, it is clear that 2-naphthol: vanillin: 4-nitroaniline / benzamide in the ratio 1:1:1.3 $\mathrm{mmol}$ is yielded a higher percentage of products both 
$A$ and $B$. The present ratio is in agreement with many reports. 2-naphthol: Aromatic aldehyde: Aromatic amine/amide in the ratio $1: 1: 1.3 \mathrm{mmol}^{21}$ and $1: 1: 1.2$ $\mathrm{mmol}^{7,15}$ were found to yield higher percentage. Variation on the yield of $A$ and $B$ is plotted against concentration of 4-nitroaniline/ benzamide shown (seeFig. 1 and 2).

The optimization study of tannic acid is also done by repeating the experiment with different amount of tannic acid were the reactants taken in the ratio $1: 1: 1.3 \mathrm{mmol}$. Table 3 shows the yield of compounds $A$ and $B$ when different concentrations of tannic acid were used.Graphs were plotted with amount of catalyst against yield of products and compared the results (SeeFig 3 and 4) shows the variation in the yield of products with different concentrations of catalyst, tannic acid.

From this, $0.02 \mathrm{mmol}$ of tannic acid yielded maximum of product $A$ and $0.03 \mathrm{mmol}$ of tannic acid gave maximum amount of product $B$ when the ratio<smiles>COc1cc(C(NC(=O)c2ccccc2)c2c(O)ccc3ccccc23)ccc1O</smiles>

\section{Product B}

Fig 6: Structure and IUPAC name of product B.1-[(4-hydroxy-3-methoxyphenyl) (phenylamido)] methyl naphthalene-2-ol of reactants taken in both reactions as $1: 1: 1.3 \mathrm{mmol}$ (Table 4). The result is agreeing with the synthesis of derivatives of amidoalkyl naphthols using tannic acid catalyst ${ }^{15}$. $0.02 \mathrm{mmol}$ and $0.03 \mathrm{mmol}$ of tannic acid in reactions to get products $A$ and $B$ respectively gave maximum amounts of products and no more addition or removal of catalyst can cause increase in the yield of products.

Hence the reactants 2-naphthol: vanillin: p-nitroaniline $(A)$ /benzamide $(B)$ in the ratio 1:1:1.3 mmol with $0.02 \mathrm{mmol}$ tannic acid to product $A$ and $0.03 \mathrm{mmol}$ of tannic acid to product $B$ is used throughout the synthesis of compounds in different methods. The results obtained from above methods and yield of final products $A$ and $B$ are tabulated. Table 4 shows the yield of products $A$ and $B$ by following various methods.

Hence synthesis in oil bath is the best method to get higher percentage of product $A$ and B. This method gave product A with $90.01 \%$ and product B with $92.10 \%$ of yield. Many experiments shown similar results in the synthesis of different derivatives belong to this category ${ }^{7}$.Also, the result agreed with the report of ${ }^{10,22}$ etc.

Microwave irradiation of synthesis of products is in competitive with yield obtained by oil bath. The present catalyst, naturally occurring tannic acid is good enough to synthesize aminoalkyl as well as amidoalkylnaphthol (A and B) with $87-89 \%$ yields. This is comparable with the works done by Zare et $\mathrm{al}^{8}$ who prepared amidoalkyl derivatives in $85-90 \%$ yield with a synthetic ionic liquid 1-butyl3-methylimidazolium bromide [Bmim] Br). The conditions they used were $800 \mathrm{~W}$ and $130^{\circ} \mathrm{C}$. In the present reaction conditions optimized is $480 \mathrm{~W}$ and $120^{\circ} \mathrm{C}$ to get higher percentage of products.

Table 4: Yield of Products a and b from Various Methods of Synthesis

\begin{tabular}{lccc}
\hline No. & Method of synthesis & \multicolumn{2}{c}{ Yield (\%) } \\
\cline { 3 - 4 } & & Product A (Aminoalkylnaphthol) & Product B (Amidoalkylnaphthol) \\
\hline I & Oil bath & 90.01 & 92.1 \\
II & Microwave irradiation & 87.32 & 89.99 \\
III & Hot plate with magnetic stirrer & 82.045 & 83.3 \\
IV & Grindstone method & 77.921 & 36.32 \\
V & Normal refluxing & 52.76 & 54.367 \\
\hline
\end{tabular}


Synthesis using direct hot plate with magnetic stirrer is moderately efficient in the synthesis of products $A$ and $B$ when compared to microwave and oil bath heating. This trend is also reported by Rajesh et $\mathrm{al}^{15}$. They explained that, low yield of products is due to non-uniform heating which may lead to the formation of undesired products. Grindstone chemistry method is applicable for the synthesis of compound A (77.92\%) but ${ }^{17}$ reported that the synthesis of aminoalkylnaphthol derivatives with $95 \%$ of yield can be obtained by using the same method. Our experiments cannot achieve much higher yields of compound $A$ by following the same method. In case of compound $B$, only $36.32 \%$ of product is separated. That shows the method fails in the synthesis of amidoalkylnaphthol, compound B.

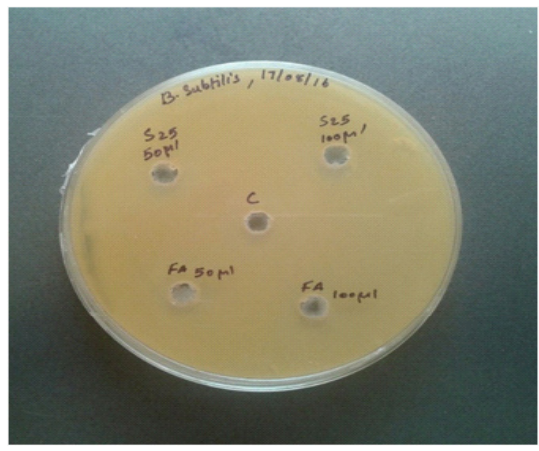

Fig 7: Zone inhibition test of compounds A and $B$ in a medium of Bacillus subtilis. Code $F_{4}$ stands for compound $A$ and code $S_{25}$ indicates compound B
To show the advantages, efficiency and applicability of tannic acid, turn over frequency (TOF) of the catalyst is calculated in different methods and compared with already reported works. Table 6 shows the TOF of tannic acid in various methods. Values of turn over frequency is tabulated in Table 5. Higher the TOF value, greater will be the efficiency of the catalyst. TOF calculations shows that, tannic acid is more efficient in the synthesis of products by green paths such as synthesis using oil bath and using microwave irradiation. TOF of tannic acid is higher for the synthesis of Product A (12.855 min-1).

\section{Mechanism of formation of product $\mathbf{A}$}

Aldehyde group of vanillin is attacked by the Lewis acid, tannic acid to facilitate the addition of vanillin to $\alpha$ position adjacent to the carbon which contains - $\mathrm{OH}$ group. Then the intermediate complex eliminates $-\mathrm{OH}$ and $\mathrm{a}-\mathrm{H}$ as a water molecule to form a double bond between 2-naphthol and - $\mathrm{CH}$ group of vanillin. This intermediate formed by the nucleophilic addition of 2-naphthol with vanillin belongs to a type of compound known as ortho-quinonemethides ${ }^{7,13}$. When 4-nitroaniline added to this intermediate in presence of tannic acid, Michael addition occurs and product $A$ is obtained. This mechanism is assigned with the help of many experiments on the synthesis of similar type of compounds which are already reported ${ }^{7,15,17}$. IUPAC name and structure of product $A$ is given (seeFig 5).

\section{Mechanism of reaction for the formation of product B}

Aldehyde group of vanillin is attacked by the Lewis acid, tannic acid to facilitate the addition

Table 5: Determination of turn over frequency of tannic acid in different methods of synthesis

\begin{tabular}{|c|c|c|c|c|c|c|c|c|c|}
\hline \multirow[t]{2}{*}{ No. } & \multirow[t]{2}{*}{$\begin{array}{l}\text { Method of C } \\
\text { synthesis } \tan \end{array}$} & \multicolumn{2}{|c|}{$\begin{array}{l}\text { Concentration of } \\
\text { tannic acid ( } \mathrm{mol} \%)\end{array}$} & \multicolumn{2}{|c|}{$\begin{array}{l}\text { Reaction time } \\
\quad(\min )\end{array}$} & \multicolumn{2}{|c|}{$\begin{array}{c}\text { Yield of } \\
\text { product (\%) }\end{array}$} & \multicolumn{2}{|c|}{ TOF $\left(\mathrm{min}^{-1}\right)$} \\
\hline & & A & B & A & B & A & B & A & B \\
\hline I & Oil bath & 2 & 3 & 12 & 14 & 90.01 & 92.1 & 3.750 & 2.193 \\
\hline II & Microwave irradiation & 2 & 3 & 3.5 & 4 & 87.32 & 89.99 & 12.855 & 7.499 \\
\hline III & $\begin{array}{l}\text { Hot plate with } \\
\text { magnetic stirrer }\end{array}$ & 2 & 3 & 23.5 & 24 & 82.045 & 83.3 & 1.7456 & 1.1563 \\
\hline IV & Grindstone method & 2 & 3 & 10 & 14 & 77.921 & 36.32 & 3.896 & 0.8647 \\
\hline V & Normal refluxing & 2 & 3 & 70 & 85 & 52.76 & 53.36 & 0.3768 & 0.3138 \\
\hline
\end{tabular}


of vanillin to $\alpha$ position adjacent to the carbon which contains - $\mathrm{OH}$ group. Then the intermediate complex eliminates $-\mathrm{OH}$ and $\mathrm{a}-\mathrm{H}$ as a water molecule to form a double bond between 2-naphthol and $-\mathrm{CH}$ group of vanillin. This intermediate formed by the nucleophilicaddition of 2-naphthol with vanillin belongs to a type of compound known as ortho-quinonemethides. When benzamide added to this intermediate in presence of tannic acid, Michael addition occurs and product $B$ is obtained. This mechanism is assigned with the help of many published reports on the synthesis of similar type of compounds ${ }^{7,13,15}$. IUPAC name and structure of product $B$ is given (seeFig 5 ).

\section{Anti-bacterial activity of products}

Products $A$ and $B$ is tested for antibacterial activity against Bacillus subtilis. Compounds are introduced in concentrations $50 / \mathrm{l}$ and $100 / \mathrm{l}$. It is found that both compounds shows negligible inhibition against the growth of this bacterium. Inhibition zone is measured only $1 \mathrm{~mm}$ range in diameter and it cannot be considered as an effective activity. Some modifications structure of compounds or in type of bacteria, storage conditions, growth of bacterium, size of plate etc may lead to better results. The petri plate showing this experiment is shown (seeFig 6).

\section{Characterization data:Product $\mathbf{A}$}

UV-VIS: $\lambda_{\max }=1232 \mathrm{~nm}(44.327 \%)$

$\operatorname{IR}(\mathrm{KBr})\left(v_{\text {max }} / \mathrm{cm}^{-1}\right): 3062.96,3022.45,2974$, 2947, 2848 (Ar-C-H and Ar-OH str), 817.8,761,729 (out of plane Ar-C-H bending), 1508-1458 (Ar-C=C str), 3404 (-N-H str), 1390-1377 (Ar-C$\mathrm{NO}_{2}$ str), 1591 (out of plane $-\mathrm{N}-\mathrm{H}$ bending), 30003500 (broad, hydrogen bonded -O-H ), 1508-1380 (deformation of -OH), 1271 (C-O-C str), 1377 (-C-O).

${ }^{1} \mathrm{H}$ NMR $\left(\mathrm{CDCl}_{3}, 400 \mathrm{MHz}\right)(\delta / \mathrm{ppm}): 3.861$ (s, 3H), $6.042(\mathrm{~s}, 1 \mathrm{H}), 6.953-6.974(\mathrm{~d}, 1 \mathrm{H}), 7.689-$ 7.026 (m, Ar H s), 9.744 (s, 1H).

${ }^{13} \mathrm{C}$ NMR (DMSO, 400MHz) ( $\left.\delta / \mathrm{ppm}\right)$ : $153.618,134.654,128.89,126.47,126.357$, $123.525,117.871,109.499,151.832,134.654$, $126.357,151.832,147.201,56.130,114.442$ and 109.34 .
GC-MS (m/z): $415.1407(\mathrm{M}+), 301.08443$, 279.10241 (base peak), 247.07607, 219.08113, 208.00493, 175.97863 and 151.09348.

Product B

$$
\text { UV-VIS: } \lambda_{\max }=1234 \mathrm{~nm}(64.018 \%)
$$

$\operatorname{IR}(\mathrm{KBr})\left(v_{\max } / \mathrm{cm}^{-1}\right):$ 3064, 3032, 2976, 2947 and 2848 (Ar-C-H str), 856, 819,779 (out of plane ArC-H bending), 1587 and 1512 ( $\mathrm{Ar}-\mathrm{C}=\mathrm{C}$ str), 3365.78 and $3296(-\mathrm{N}-\mathrm{H}$ str), 1587 and 1512 (out of plane $-\mathrm{N}-\mathrm{H}$ bending), 3498-3033 (broad, hydrogen bonded $-\mathrm{O}-\mathrm{H}$ str and $-\mathrm{N}-\mathrm{H}$ str), 1292 and 1269 (-C-O-C str), 1377 (-C-O str), 1664 (-C=O str).

${ }^{1} \mathrm{H}$ NMR $\left(\mathrm{CDCl}_{3}, 400 \mathrm{MHz}\right)(\mathrm{ä} / \mathrm{ppm}): 3.5$ (s, 3H), $2.5(\mathrm{~s}, 1 \mathrm{H})$, 7. 088-7.082 (d, 1H), 7.689-7.026 (m, Ar H s), $9.783(\mathrm{~s}, 1 \mathrm{H})$.

${ }^{13} \mathrm{C}$ NMR (DMSO, 400MHz) ( $\left.\delta / \mathrm{ppm}\right): 55.563$, $108.604,110.695,115.369,118.559,122.604$, $125.935,126.074,127.494,153.009,134.554$, $128.678,167.898,153.009,148.137,153.009$ and 155.219 .

GC-MS (m/z): $398.0802(\mathrm{M}+), 387.08802$, $376.13178,353.12717,301.08449,279.10246$, 247.07602, 232.07394, 219.08174, 175.97959, 167.07083 and 151.03954 (base peak).

Amidoalkylnaphthols and aminoalkyl naphthols are important compounds in the field of pharmaceutical chemistry. They may act as starting compounds in the synthesis of many valuable drugs which may cure several diseases such as Parkinson's disease, hypertension etc. Through this study, we successfully synthesized an aminoalkylnaphthol (product A) from vanillin, 2-naphthol, and 4-nitroaniline and an amidoalkylnaphthol (product B) from vanillin, 2-naphthol and benzamide by various green chemical approaches of synthesis such as microwave irradiation, oil bath, hot plate with magnetic stirrer and Grindstone chemistry method using tannic acid as catalyst. The yield of products are compared with products obtained from normal heating of reactants under reflux using solvent and found that, heating in oil bath is the best method to get maximum products. Oil bath affords $90.01 \%$ and $92.1 \%$ of products. Microwave irradiation is also good for getting products with yield 87 and $89 \%$. 
Grindstone chemistry method is only applicable in the synthesis of aminoalkylnaphthol. Optimization study of reactants and tannic acid are done. It is found that, for synthesizing both type of compounds with maximum yield, reactants should be in the ratio $1: 1: 1.3 \mathrm{mmol}$ where the third constituent is 4-nitroaniline or benzamide. $0.02 \mathrm{mmol}$ of tannic acid is effective in the case of formation of product A (aminoalkylnaphthol) and for product B it is 0.03 $\mathrm{mmol}$ of tannic acid. The reactants and products are well separated in TLC when the solvent system is a mixture of hexane and ethyl acetate in the ratio 4:1. Products are isolated from crude sample by column chromatography using 1:1 hexane ethyl acetate mixture as eluting solvent. The isolated compounds are tested for their antibacterial activity against Bacillus Subtilis and found that they shows very little effect on the inhibition against bacterial growth rate. Many factors such as structure of compound, concentration of compounds applied, $\mathrm{PH}$ conditions of the culture medium, bacterial growth concentration, size of the petri plate, storage conditions etc may affect the inhibition rate. Hence we hope in future studies, these compounds may exhibit higher efficiency of inhibition against this bacterium by varying these factors or they may exhibit inhibition against growth of other bacteria and also anti-fungal activities.

\section{REFERENCES}

1. Shen, G.S.; Layer, R.T.; and Mabe R.T.;Drug Discov., Today. 2000, 5, 98-106.

2. Damodiran, N.;PanneerSelvam;Paramasivan T.;Perumal,; Tetrahedron Lett 2009, 54745478.

3. Basappa,; Sengottuvelan Murugan,; Chandagirikoppal, V.;Kavitha,; Anurag Purushothaman,;Kottayath, G. Nevin,; Kazuyuki Sugahara,; Kanchugarakoppal, S.;Rangappa,; Cancer Lett 2010, 231-243.

4. Aitken,;. AitkenK. M.; University of St. Andrews, St. Andrews, UK, "1, 4-Oxazines and their Benzo Derivatives", Comprehensive Heterocyclic Chemistry III, 2008, 8, 373459.

5. SrinivasKantevari,;Srinivasu V.N.;Vu ppalapati,;LingaiahNagarapu,;Catalysis Communications, 2007, 266, 109.

6. Selvam, N. P.; and Perumal, P T.; Tetrahedron Letters, 2006,7481-7483.

7. Deepali, A.;Kotadia,;Saurabh, S.;Soni,; J. Mol. A. Catal: Chem,2012, 353-354.

8. Zare, A.;Hasaninejad,;SalimiBeni, A.;MoosaviZare, A.R.;Merajoddin,M.;Kamali,E.;Akba ri-Seddigh,M.; Parsee, Z.; Scientialranica, 2011, 18, 433-438.

9. RaminGhorbani-Vaghei,;Seyedeh Mina Malaekehpour,;Cent. Eur. J. Chem., 2010,8, 1086-1089.

10. AbolghasemDavoodnia,;RahilMahjoobin,;Nil oofarTavakoliHoseini ,;Chin. J. Catal., 2014, 35, 490-495.
11. SamadKhaksar,;RoshanakNajafi,;SeyedMojt abaOstad,;MahgolTajbakhsh,; World App. Sci. J., 2012, 20, 656-660.

12. Ali Dorehgiraee,;HojatollahKhabazzadeh,;Ka zemSaidi;ARKIVOC, 2009, 7, 303-310.

13. ZahedKarimi-Jaberi,;HadiFakhraei,;Bull. Chem. Soc. Ethiop., 2012, 26, 473-478.

14. Hassan Moghanian,;Satter Ebrahimi,;2014, 18, 165-168.

15. Rajesh. Singh,;RobithaDuvedi,;Arabian journal of chemistry,2014.

16. Nana V. Shitole,;Balasahe V. Shitole,;Gopal K. Kakdeb,;Murlidhar S. Shingare,;Orbital: The Electronic Journal of Chemistry, 2013, 5.

17. Madan Mohan M, C.;Santosh and Radhaiah, A.;AdikaviNannaya,; University, Int.J.Curr. Microbiol.App.Sci, 2015, 4, 1000-1008.

18. VikasVerma, Kuldeep Singh, Devinder Kumar, Thomas M. Klapotke, Jorg Stierstorfer, BalasubramanianNarasimhan , AsifKhurshidQazi, Abid Hamid , SundeepJaglan, European Journal of Medicinal Chemistry, 2012, 195-202.

19. Leila Zamani, KamiarZomorodian, B i b i Fath e m e i M i r jalin i, SoghraKhabnadidehJournal of Pharmaceutical and Scientific Innovation, 2014

20. Siddique Akbar M. K Ansari,Jayprakash M. Sangshetti, Nagnath D. Kokare, Pravin S. Wakte, Devanand V. Shinde, Indian J. Chem. Technol., 2010, 17, 71-73. 
21. NourallahHazeri, MalekTaherMaghsoodlou, Sayyed Mustafa Habibi-khorassani, JasemAboonajmi and MohyeddinSafarzaei, Chem. Sci. Trans., 2013, 2, S330-S336.
22. Hamid Reza Shaterian, HosseinYarahmadi and MajidGhashang, Bioorg. Med. Chem. Lett., 2008,33, 788-792. 